Proceedings

\title{
Development of a Nanodroplet Formulation for Triggered Release of BIO for Bone Fracture Healing ${ }^{\dagger}$
}

\author{
Jonathan P. May 1,2,3, Anastasia Polydorou 1,2, Sara Ferri 1,2,3, Qiang Wu ${ }^{4}$, Eleanor Stride ${ }^{4, *}$, Dario Carugo 1,3,* and \\ Nicholas D. Evans 1,2,*
}

Citation: May, J.P.; Polydorou, A.; Ferri, S.; Wu, Q.; Stride, E.; Carugo, D.; Evans, N.D. Development of a Nanodroplet Formulation for Triggered Release of BIO for Bone Fracture Healing. Proceedings 2021, 78, 43. https://doi.org/10.3390/ IECP2020-08803

Published: 1 December 2020

Publisher's Note: MDPI stays neutral with regard to jurisdictional claims in published maps and institutional affiliations.

Copyright: $(2020$ by the authors. Licensee MDPI, Basel, Switzerland. This article is an open access article distributed under the terms and conditions of the Creative Commons Attribution (CC BY) license (http://creativecommons.org/licenses/by/4.0/).
1 Bioengineering Group, Faculty of Engineering and Physical Sciences, University of Southampton, Southampton SO17 1BJ, UK; j.p.may@soton.ac.uk (J.P.M.); a.e.polydorou@soton.ac.uk (A.P.); s.ferri@soton.ac.uk (S.F.)

2 Bone and Joint Research Group, Human Development and Health, Faculty of Medicine, University of Southampton, Southampton General Hospital, Southampton SO16 6YD, UK

3 Department of Pharmaceutics, UCL School of Pharmacy, University College London, London WC1N 1AX, UK

4 Institute of Biomedical Engineering, Old Road Campus Research Building, University of Oxford, Oxford OX3 7DQ, UK; qiang.wu@eng.ox.ac.uk

* Correspondence: eleanor.stride@eng.ox.ac.uk (E.S.); d.carugo@ucl.ac.uk (D.C.); n.d.evans@soton.ac.uk (N.D.E.)

+ Presented at the 1st International Electronic Conference on Pharmaceutics, 1-15 December 2020; Available online: https://iecp2020.sciforum.net/.

\begin{abstract}
Impaired fracture healing impacts patients' quality of life and imposes a financial burden on healthcare services. Up to $10 \%$ of bone fractures result in delayed/non-union fractures, for which new treatments are urgently required. However, systemic delivery of bone anabolic molecules is often sub-optimal and can lead to significant side effects. In this study, we developed ultrasound (US) responsive nano-sized vehicles in the form of perfluorocarbon nanodroplets (NDs), as a means of targeting delivery of drugs to localised tissues. We tested the hypothesis that NDs could stably encapsulate BIO (GSK-3 $\beta$ inhibitor), which could then be released upon US stimulation to activate Wnt signalling and induce ossification. NDs $(\sim 280 \mathrm{~nm})$ were prepared from phospholipids and liquid perfluorocarbon and their stability and drug loading was studied by NTA (Nano Tracking Analysis) and HPLC. ND cytotoxicity was assessed in patient-derived bone marrow stromal cells (BMSCs) with Alamar Blue (24 h), and in vitro bioactivity of BIO-NDs was evaluated in a 3T3 Wntpathway reporter cell line with luciferase readout. To investigate the acoustic behaviour of NDs, $2 \%$ agarose (LM) containing NDs was injected into a bespoke bone fracture model (Sawbones) of various geometries and stimulated by US ( $1 \mathrm{MHz}, 5 \%$ duty cycle, $1 \mathrm{MPa}, 30 \mathrm{~s})$, allowing the simultaneous capture of optical images and acoustic emissions. Femoral bone hole defects (1-2 mm) were made in WT-MF1 mice (age: 8-12 wks) and DiR-labelled NDs (100 $\mu \mathrm{L}, 109 \mathrm{NDs} / \mathrm{mL}$, i.v.) were injected post-fracture to determine biodistribution by IVIS imaging. NDs were stable $\left(4\right.$ and $\left.37{ }^{\circ} \mathrm{C}\right)$ and retained $>90 \%$ BIO until US was applied, which caused $\sim 100 \%$ release. ND exposure up to a concentration of $109 \mathrm{NDs} / \mathrm{mL}$ showed no cytotoxicity (24 h). BIO-loaded NDs induced Wnt pathway activation in a dose dependent manner. Biodistribution of DiR-NDs in a femoral bone hole defect model in mice demonstrated increased localisation at the fracture site ( 2 -fold relative to that found in healthy mice or contralateral femurs at $48 \mathrm{~h}$ ).
\end{abstract}

Keywords: nanoparticle; phase-change nanodroplet; ultrasound; externally stimulated triggered release; bone fracture healing

Institutional Review Board Statement: The study was conducted according to the guidelines of the Declaration of Helsinki, and approved by the Institutional Review Board of the University of Southampton (P96B16FBD, 24 July 2019). 
Informed Consent Statement: Not applicable.

Data Availability Statement: Not applicable. 